\title{
Multiple positive radial solutions on annuli for nonlinear Neumann problems with large growth
}

\author{
Denis Bonheure and Enrico Serra
}

\begin{abstract}
We consider a classical semilinear elliptic equation with Neumann boundary conditions on an annulus in $\mathbf{R}^{N}$. The nonlinear term is the product of a radially symmetric coefficient with a pure power. We prove that if the power is sufficiently large, the problem admits at least three distinct positive and radial solutions. In case the coefficient is constant, we show that none of the three solutions is constant. The methods are variational and are based on the study of a suitable limit problem.
\end{abstract}

Mathematics Subject Classification (2000). 35J60, 35J20.

Keywords. Neumann problems, Large growth, Radial solutions, Multiplicity results.

\section{Introduction}

In this paper we study the existence of multiple solutions to the Neumann problem

$$
\begin{cases}-\Delta u+u=g(|x|) u^{p-1} & \text { in } \Omega \\ u>0 & \text { in } \Omega \\ \frac{\partial u}{\partial \nu}=0 & \text { on } \partial \Omega\end{cases}
$$

where $\Omega$ is an annulus in $\mathbf{R}^{N}$ :

$$
\Omega=\left\{x \in \mathbf{R}^{N}|a<| x \mid<b\right\} .
$$

We always assume that the function $g$ is nonnegative and that $p>2$. Although our results hold when $N=2$, we are mainly interested in the case $N \geq 3$.

Before describing our main results, it is interesting to compare problem (1) to one of its Dirichlet analogues, namely 


$$
\begin{cases}-\Delta u=u^{p-1} & \text { in } \Omega \\ u>0 & \text { in } \Omega \\ u=0 & \text { on } \partial \Omega,\end{cases}
$$

This problem has been widely studied on general domains (not necessarily annuli) because of its complex phenomenology concerning existence and nonexistence of solutions in dependence of the exponent $p$, of lower order perturbations, of the topology and the geometry of the domain.

It is well known for example that if $N \geq 3, \Omega$ is starshaped and $p \geq 2^{*}=$ $2 N /(N-2)$, then no solution exists. On the contrary, it has been recognized in the pioneering paper [3] that lower order perturbations of $u^{p}$ can reverse the situation when $p=2^{*}$. The same happens if the domain has nontrivial topology [1]. When $p$ is supercritical (i.e. $p>2^{*}$ ), the situation is more complex. Indeed, there are examples [10] that show that the problem may have no solutions even if the topology of the domain is nontrivial, and it is possible that also the exact values of $p$ play a role for existence. As a general fact, not much is known in the supercritical range, except for two cases. The first occurs in the presence of slightly supercritical growth, and the second when the domain possesses some symmetries, for example when it is invariant under rotations. From the point of view of this paper, the case of an annulus is particularly relevant. For the Dirichlet problem (3) when $\Omega$ is the annulus (2), a classical result by Kazdan and Warner [6] states that for every $p>2$, problem (3) admits a radial solution. This is not surprising, since the embedding of $H_{0 \text {, rad }}^{1}(\Omega)$ into $L^{p}(\Omega)$ is compact for every $p \geq 1$, and hence the radially symmetric problem can be solved with by now standard variational techniques. The result of [6] is complemented by the papers [9], according to which the radial solution is unique and [4], where the asymptotic behavior of the solution as $p \rightarrow \infty$ is described. Thus the picture concerning the solvability of the Dirichlet problem on the annulus is quite precise: there exists exactly one radial solution, independently of the size of $p$. At least the existence part of these results can be extended to equations with a radially symmetric coefficient in front of the nonlinearity, such as the right-hand-side of the equation in (1) with reasonable assumptions and without particular effort.

In passing from Dirichlet to Neumann boundary conditions, one encounters a remarkable phenomenon: existence, multiplicity and nonexistence results abound when $p \leq 2^{*}$, while are extremely scarce above the critical exponent. As far as nonexistence is concerned, the lack of results can be explained by observing that the classical tool providing nonexistence theorems, the Pohožaev identity [11], does not work well with Neumann conditions. Actually, when $g \equiv 1$ the equation in (1) admits the constant solution $u \equiv 1$ on any domain and for every $p$. On the other hand, the failure of the methods based on the Pohožaev identity in the nonautonomous case $(g \not \equiv 1)$, should stimulate intense research around supercritical Neumann problems and the scarcity of results in that direction is our main motivation.

It seems that supercritical Neumann problems have not been studied deeply so far, with two exceptions, similarly to the Dirichlet case: slightly supercritical growths and singular perturbations, areas in which the literature 
is quite rich. Few papers are devoted to the analysis of problems like (1) when $p>2^{*}$, even on annuli or balls, domains that simplify the study because they allow one to work with radial functions. If the problem is autonomous $(g \equiv 1)$, the classical paper [8] by $\mathrm{Ni}$ can be invoked to prove the existence on one positive (nonconstant) solution on a ball provided its radius is large enough. Also [7] deals with supercritical autonomous problems, providing conditions (on the radius) for existence and nonexistence of radial nonconstant solutions on balls. More recently, the paper [2] establishes an existence result for the Hénon equation $\left(g(|x|) \equiv|x|^{\alpha}, \alpha>0\right)$ on the unit ball for every $p>2$, using a shooting argument. The result of [2] has been extended and recast in a variational setting in [12], where increasing weights $g$ are considered. The main theorem in [12] proves the existence of at least one solution on any ball and for every $p>2$ by constructing a variational principle on the set of increasing functions.

If one considers annuli, the existence of one radial solution for problems even more general than (1) poses no difficulties. By this we mean that working with supercritical $p$ 's on an annulus and looking for radial solution is of course the same as dealing with the subcritical case, because of the Rellich-Sobolev embeddings. As far as we know however, no multiplicity result is known for radial solutions to problem (1) even when $p$ is "large".

The purpose of this paper is to establish a multiplicity result under general conditions on $g$. The following is our main result.

Theorem 1.1. Assume that $g \in L^{1}(a, b)$ and satisfies $g(r)>0$ a.e. in $(a, b)$. Then for every $p$ large enough, problem (1) admits at least three distinct radial solutions.

The proof of this result is based on the analysis of a suitable problem "at infinity", that in the present case is represented by the minimization of the functional $Q_{\infty}: H_{\text {rad }}^{1}(\Omega) \backslash\{0\} \rightarrow \mathbf{R}$ given by

$$
Q_{\infty}(u)=\frac{\|u\|_{H^{1}}^{2}}{\|u\|_{L^{\infty}(\Omega)}^{2}} .
$$

This functional arises when formally letting $p \rightarrow \infty$ in the Rayleigh functional associated to (1),

$$
Q_{p}(u)=\frac{\|u\|_{H^{1}}^{2}}{\left(\int_{\Omega} g(|x|)|u|^{p} d x\right)^{2 / p}} .
$$

In a very concise form, we anticipate that the functional $Q_{\infty}$ is shown to have two strict local minima on the sphere of $H_{r a d}^{1}(\Omega)$, so that the same happens for $Q_{p}$, provided one proves that $Q_{p}$ and $Q_{\infty}$ are suitably close when $p$ is large. This topological structure allows one to find a third solution by means of a minimax procedure. The main point is thus the proof of the existence of two local minima for $Q_{\infty}$, and this is carried out by studying in some detail an intermediate minimization problem depending on a parameter. More precisely, the most important role in this paper is played by the function $\varphi:[a, b] \rightarrow \mathbf{R}$ 
defined by

$$
\varphi(\rho)=\min _{\substack{u \in H_{\text {rad }}^{1}(\Omega) \\ u(\rho) \neq 0}} \frac{\|u\|_{H^{1}}^{2}}{u(\rho)^{2}} .
$$

The function $\varphi$ is shown to have two local minima at $a$ and $b$, and this is what allows us to identify two open sets in $H_{\text {rad }}^{1}(\Omega)$ where the functional $Q_{\infty}$ can be minimized, giving rise to the two aforementioned minima.

A further natural question arises when dealing with the autonomous version of problem (1), namely when $g \equiv 1$. In this case indeed one may wonder what happens of the three (obviously nonconstant, when $g \not \equiv 1$ ) solutions of Theorem 1.1. It is quite predictable that the two local minima are not constant, but it is much less clear whether the third solution, obtained by a mountain pass procedure, is not constant. The variational characterization of the third solution allows us to answer the question in the affirmative.

Theorem 1.2. For every $p$ large enough, the problem

$$
\begin{cases}-\Delta u+u=u^{p-1} & \text { in } \Omega \\ u>0 & \text { in } \Omega \\ \frac{\partial u}{\partial \nu}=0 & \text { on } \partial \Omega\end{cases}
$$

admits at least three distinct nonconstant radial solutions.

Thus problem (4) admits four positive radial solutions, three of which are not constant. We believe that this is an interesting phenomenon, also because it does not depend on the size of the annulus.

The paper is structured as follows. In Sect. 2 we introduce the function $\varphi$ and prove its principal properties. Section 3 is devoted to the analysis of the limit problem involving the functional $Q_{\infty}$. Theorem 1.1 is proved in Sect. 4, while Sect. 5 contains the proof of Theorem 1.2.

Notation. The notation we use is standard. Throughout the paper $\omega$ denotes the measure of the unit sphere in $\mathbf{R}^{N}$. The scalar product in the Sobolev space $H^{1}$ is denoted by $\langle\cdot, \cdot\rangle$. The $L^{p}$ norm of a function $u$ is written $\|u\|_{p}$. Expressions like $u\left(\rho^{+}\right)$stand for $\lim _{r \rightarrow \rho^{+}} u(r)$. For any radial function $u$, we write freely $u(x)$ or $u(r)$, with $r=|x|$.

\section{An auxiliary problem}

In this section we discuss the properties of an auxiliary problem that will turn out to be crucial for the proof of the main result.

We recall that $\Omega$ is the annulus $\left\{x \in \mathbf{R}^{N}|a<| x \mid<b\right\}$, with $N \geq 2$. We work in the space $H_{\text {rad }}^{1}(\Omega)$ of radially symmetric $H^{1}$ functions, with norm

$$
\begin{aligned}
\|u\|^{2} & =\int_{\Omega}|\nabla u(x)|^{2} d x+\int_{\Omega} u(x)^{2} d x \\
& =\omega \int_{a}^{b}\left|u^{\prime}(r)\right|^{2} r^{N-1} d r+\omega \int_{a}^{b} u(r)^{2} r^{N-1} d r .
\end{aligned}
$$


For each fixed $\rho \in[a, b]$, we define

$$
H_{\rho}=\left\{H_{\text {rad }}^{1}(\Omega) \mid u(\rho) \neq 0\right\}
$$

and we consider the functional $F: H_{\rho} \rightarrow \mathbf{R}$ defined by

$$
F(v)=\frac{\|v\|^{2}}{v(\rho)^{2}},
$$

Lemma 2.1. For every $\rho \in[a, b]$, there exists $u \in H_{\rho}$ such that

$$
F(u)=\inf _{v \in H_{\rho}} F(v)>0 .
$$

Proof. It is an immediate consequence of the fact that the embedding of $H_{\text {rad }}^{1}(\Omega)$ into $C(\bar{\Omega})$ is compact.

Definition 2.2. We define the function $\varphi:[a, b] \rightarrow \mathbf{R}$ by

$$
\varphi(\rho)=\min _{v \in H_{\rho}} F(v) .
$$

Thus, for every $\rho \in[a, b]$, the function $\varphi(\rho)$ is well defined and strictly positive. We now study some properties of $\varphi$.

Let $u \in H_{\rho}$ be a minimizer for $F$. Then

$$
F^{\prime}(u) v=0 \quad \forall v \in H_{\text {rad }}^{1}(\Omega),
$$

which reads

$$
\langle u, v\rangle-\frac{\|u\|^{2}}{u(\rho)} v(\rho)=0 \quad \forall v \in H_{r a d}^{1}(\Omega) .
$$

Assume first that $\rho \in(a, b)$, and set

$$
\Omega^{-}=\{x \in \Omega|a<| x \mid<\rho\}, \quad \Omega^{+}=\{x \in \Omega|\rho<| x \mid<b\} .
$$

Then, choosing $v \in H_{\text {rad }}^{1}(\Omega)$ such that $v(\rho)=0$, one easily sees that $u$ solves

$$
\begin{cases}-\Delta u+u=0 & \text { in } \Omega^{-} \cup \Omega^{+} \\ \frac{\partial u}{\partial \nu}=0 & \text { on } \partial \Omega \\ u\left(\rho^{-}\right)=u\left(\rho^{+}\right) & \end{cases}
$$

Remark 2.3. If one normalizes functions in $H_{\rho}$ by $v(\rho)=1$, then the minimization problem (5) has a unique solution. Indeed if $u$ and $v$ are two solutions, then $w=u-v$ solves

$$
\begin{cases}-\Delta w+w=0 & \text { in } \Omega^{-} \\ \frac{\partial w}{\partial \nu}=0 & \text { on }|x|=a \\ w=0 & \text { on }|x|=\rho,\end{cases}
$$

and similarly on $\Omega^{+}$. Multiplying the equation by $w$ and integrating immediately gives $w \equiv 0$ in $\Omega^{-}$, and the same in $\Omega^{+}$. 
The unique (normalized) solution $u$ of Problem (5) is positive and smooth except at $|x|=\rho$. Since $u$ is radial, the equation in (6) reads

$$
\left(r^{N-1} u^{\prime}(r)\right)^{\prime}=r^{N-1} u(r) \quad \forall r \in(a, \rho) \cup(\rho, b),
$$

from which one easily sees that $\rho$ is the unique absolute maximum of $u$ on $[a, b]$ and that $u$ is strictly increasing before $\rho$ and strictly decreasing after $\rho$.

For further reference we notice that integrating $(8)$ on $(a, \rho)$ and on $(\rho, b)$ yields

$$
u^{\prime}\left(\rho^{-}\right)=\frac{1}{\rho^{N-1}} \int_{a}^{\rho} u(r) r^{N-1} d r \quad \text { and } \quad u^{\prime}\left(\rho^{+}\right)=-\frac{1}{\rho^{N-1}} \int_{\rho}^{b} u(r) r^{N-1} d r .
$$

We complete the description of the basic properties of $u$ by examining the cases $\rho=a$ and $\rho=b$; in these cases the situation is simpler. Indeed, for example for $\rho=a$, there is a unique minimizer $u$ normalized by $u(a)=1$, and it satisfies

$$
\begin{cases}-\Delta u+u=0 & \text { in } \Omega \\ u=1 & \text { on }|x|=a \\ \frac{\partial u}{\partial \nu}=0 & \text { on }|x|=b .\end{cases}
$$

It is easy to see that $u$ is positive, smooth and strictly radially decreasing. The solution corresponding to $\rho=b$ is positive, smooth and strictly radially increasing.

We now intend to study some properties of the function $\varphi$ introduced in Definition 2.2. To this aim we denote by $u_{\rho}:[a, b] \rightarrow \mathbf{R}$ the unique solution of the minimization problem $(5)$, normalized by $u_{\rho}(\rho)=1$. Thus,

$$
\left\{\begin{array}{l}
-\left(r^{N-1} u_{\rho}^{\prime}(r)\right)^{\prime}+r^{N-1} u_{\rho}(r)=0 \quad \text { in }(a, \rho) \text { if } \rho>a \\
u_{\rho}^{\prime}(a)=0 \\
u_{\rho}(\rho)=1
\end{array}\right.
$$

and

$$
\left\{\begin{array}{l}
-\left(r^{N-1} u_{\rho}^{\prime}(r)\right)^{\prime}+r^{N-1} u_{\rho}(r)=0 \quad \text { in }(\rho, b) \text { if } \rho<b \\
u_{\rho}^{\prime}(b)=0 \\
u_{\rho}(\rho)=1
\end{array}\right.
$$

Remark 2.4. Applying standard regularity theory for ODEs one sees that the restriction of $u_{\rho}$ to $[a, \rho]$ or $[\rho, b]$ is smooth (say $\left.C^{2}\right)$ in $(r, \rho)$. One can also check this by noting that the radial solutions of $-\Delta u+u=0$ can be explicitly written down in terms of modified Bessel functions.

Since by definition

$$
\varphi(\rho)=\left\|u_{\rho}\right\|^{2}=\omega \int_{a}^{\rho}\left(\left|u_{\rho}^{\prime}\right|^{2}+u_{\rho}^{2}\right) r^{N-1} d r+\omega \int_{\rho}^{b}\left(\left|u_{\rho}^{\prime}\right|^{2}+u_{\rho}^{2}\right) r^{N-1} d r,
$$

we see that $\varphi$ is continuous on $[a, b]$ and differentiable on $(a, b)$.

We now state one of the main properties of $\varphi$.

Proposition 2.5. The points $a$ and $b$ are local minima for $\varphi$. 
Proof. We compute the derivative of the function $\varphi$. To this aim, we write $\varphi(\rho)=\omega f(\rho)+\omega h(\rho):=\omega \int_{a}^{\rho}\left(\left|u_{\rho}^{\prime}\right|^{2}+u_{\rho}^{2}\right) r^{N-1} d r+\omega \int_{\rho}^{b}\left(\left|u_{\rho}^{\prime}\right|^{2}+u_{\rho}^{2}\right) r^{N-1} d r$, and we carry out the computations for $f$ only. Now, for every $\rho \in(a, b)$, we have

$$
\begin{aligned}
f^{\prime}(\rho)= & \left(u_{\rho}^{\prime}\left(\rho^{-}\right)^{2}+u_{\rho}(\rho)^{2}\right) \rho^{N-1}+2 \int_{a}^{\rho} u_{\rho}^{\prime}(r) \frac{\partial u_{\rho}^{\prime}}{\partial \rho}(r) r^{N-1} d r \\
& +2 \int_{a}^{\rho} u_{\rho}(r) \frac{\partial u_{\rho}}{\partial \rho}(r) r^{N-1} d r \\
= & \left(u_{\rho}^{\prime}\left(\rho^{-}\right)^{2}+1\right) \rho^{N-1}+2 u_{\rho}^{\prime}\left(\rho^{-}\right) \frac{\partial u_{\rho}}{\partial \rho}\left(\rho^{-}\right) \rho^{N-1} \\
& -2 \int_{a}^{\rho}\left(\left(r^{N-1} u_{\rho}^{\prime}(r)\right)^{\prime}-r^{N-1} u_{\rho}(r)\right) \frac{\partial u_{\rho}}{\partial \rho}(r) d r \\
= & \rho^{N-1}\left(u_{\rho}^{\prime}\left(\rho^{-}\right)^{2}+1+2 u_{\rho}^{\prime}\left(\rho^{-}\right) \frac{\partial u_{\rho}}{\partial \rho}\left(\rho^{-}\right)\right)
\end{aligned}
$$

because $u_{\rho}$ solves (11).

In the same way one checks that

$$
h^{\prime}(\rho)=-\rho^{N-1}\left(u_{\rho}^{\prime}\left(\rho^{+}\right)^{2}+1+2 u_{\rho}^{\prime}\left(\rho^{+}\right) \frac{\partial u_{\rho}}{\partial \rho}\left(\rho^{+}\right)\right),
$$

so that

$$
\begin{aligned}
\varphi^{\prime}(\rho)= & \omega \rho^{N-1}\left(u_{\rho}^{\prime}\left(\rho^{-}\right)^{2}+2 u_{\rho}^{\prime}\left(\rho^{-}\right) \frac{\partial u_{\rho}}{\partial \rho}\left(\rho^{-}\right)\right. \\
& \left.-u_{\rho}^{\prime}\left(\rho^{+}\right)^{2}-2 u_{\rho}^{\prime}\left(\rho^{+}\right) \frac{\partial u_{\rho}}{\partial \rho}\left(\rho^{+}\right)\right) .
\end{aligned}
$$

Since $u_{\rho}\left(\rho^{ \pm}\right)=1$ for all $\rho$, we have

$$
\frac{\partial u_{\rho}}{\partial \rho}\left(\rho^{-}\right)+u_{\rho}^{\prime}\left(\rho^{-}\right)=0 \quad \text { and } \quad \frac{\partial u_{\rho}}{\partial \rho}\left(\rho^{+}\right)+u_{\rho}^{\prime}\left(\rho^{+}\right)=0,
$$

and inserting these in (14) yields

$$
\varphi^{\prime}(\rho)=\omega \rho^{N-1}\left(u_{\rho}^{\prime}\left(\rho^{+}\right)^{2}-u_{\rho}^{\prime}\left(\rho^{-}\right)^{2}\right) .
$$

If we recall (9), this can be written

$$
\varphi^{\prime}(\rho)=\frac{\omega}{\rho^{N-1}}\left(\left(\int_{\rho}^{b} u(r) r^{N-1} d r\right)^{2}-\left(\int_{a}^{\rho} u(r) r^{N-1} d r\right)^{2}\right) .
$$

As this holds for every $\rho \in(a, b)$ and $\varphi$ is continuous on $[a, b]$, we can let $\rho$ tend to $a$ or to $b$. We obtain

$$
\varphi^{\prime}\left(a^{+}\right)=\frac{\omega}{a^{N-1}}\left(\int_{a}^{b} u(r) r^{N-1} d r\right)^{2}>0
$$


and

$$
\varphi^{\prime}\left(b^{-}\right)=-\frac{\omega}{b^{N-1}}\left(\int_{a}^{b} u(r) r^{N-1} d r\right)^{2}<0 .
$$

Then it is clear that $a$ and $b$ are strict local minima for $\varphi$.

In order to collect more properties of $\varphi$ we need the following lemmas.

Lemma 2.6. Let $u:[a, b] \rightarrow \mathbf{R}$ be integrable, positive and increasing, and let $\hat{u}:[a, b] \rightarrow \mathbf{R}$ be defined by $\hat{u}(r)=u(a+b-r)$. Then

$$
\int_{a}^{b} \hat{u}(r) r^{N-1} d r<\int_{a}^{b} u(r) r^{N-1} d r .
$$

Proof. Set

$$
\begin{aligned}
I & =\int_{a}^{b} u(r) r^{N-1} d r-\int_{a}^{b} \hat{u}(r) r^{N-1} d r \\
& =\int_{a}^{b} u(r) r^{N-1} d r-\int_{a}^{b} u(r)(a+b-r)^{N-1} d r \\
& =\int_{a}^{b} u(r)\left(r^{N-1}-(a+b-r)^{N-1}\right) d r .
\end{aligned}
$$

Note that $r^{N-1} \geq(a+b-r)^{N-1}$ if and only if $r \geq(a+b) / 2$. Since $u$ is increasing, we have

$$
\begin{aligned}
I= & \int_{a}^{\frac{a+b}{2}} u(r)\left(r^{N-1}-(a+b-r)^{N-1}\right) d r \\
& +\int_{\frac{a+b}{2}}^{b} u(r)\left(r^{N-1}-(a+b-r)^{N-1}\right) d r \\
\geq & u\left(\frac{a+b}{2}\right) \int_{a}^{b}\left(r^{N-1}-(a+b-r)^{N-1}\right) d r=\frac{2}{N}\left(b^{N}-a^{N}\right) u\left(\frac{a+b}{2}\right)>0 .
\end{aligned}
$$

Lemma 2.7. Let $u$ be any function in $H_{b}$ such that

$$
\varphi(b)=\frac{\|u\|^{2}}{u(b)^{2}} \text {. }
$$

Then $u$ is convex on $[a, b]$.

Proof. The function $u$ satisfies

$$
\left(r^{N-1} u^{\prime}(r)\right)^{\prime}=r^{N-1} u(r) \quad \forall r \in(a, b),
$$

and $u^{\prime}(a)=0$. Moreover, $u$ is strictly increasing. Integrating the preceding equation yields

$$
u^{\prime}(r)=\frac{1}{r^{N-1}} \int_{a}^{r} u(s) s^{N-1} d s
$$


so that

$$
u^{\prime \prime}(r)=\frac{1-N}{r^{N}} \int_{a}^{r} u(s) s^{N-1} d s+u(r) .
$$

Now on $[a, r]$ we have $u(s) \leq u(r)$, and hence

$$
\begin{aligned}
u^{\prime \prime}(r) & \geq \frac{1-N}{r^{N}} u(r) \int_{a}^{r} s^{N-1} d s+u(r)=\frac{1-N}{r^{N}} u(r)\left(\frac{r^{N}}{N}-\frac{a^{N}}{N}\right)+u(r) \\
& =\frac{1}{N} u(r)+\left(1-\frac{1}{N}\right) \frac{a^{N}}{r^{N}} u(r)>0,
\end{aligned}
$$

which shows that $u$ is convex.

We can now prove a further qualitative property of the function $\varphi$.

Proposition 2.8. There results $\varphi(a)<\varphi(b)$.

Proof. Let $u$ be such that

$$
\varphi(b)=\frac{\|u\|^{2}}{u(b)^{2}},
$$

and define, for $r \in[a, b]$,

$$
\hat{u}(r)=u(a+b-r) .
$$

By the preceding lemma, the positive function $r \mapsto u^{\prime}(r)^{2}+u(r)^{2}$ is increasing, and hence, by Lemma 2.6,

$$
\begin{aligned}
\|u\|^{2} & =\omega \int_{a}^{b}\left(u^{\prime}(r)^{2}+u(r)^{2}\right) r^{N-1} d r \\
& >\omega \int_{a}^{b}\left(\hat{u}^{\prime}(r)^{2}+\hat{u}(r)^{2}\right) r^{N-1} d r=\|\hat{u}\|^{2} .
\end{aligned}
$$

Since $\hat{u}(a)=u(b)$, we have

$$
\varphi(a) \leq \frac{\|\hat{u}\|^{2}}{\hat{u}(a)^{2}}<\frac{\|u\|^{2}}{u(b)}=\varphi(b) .
$$

\section{Analysis of a limit problem}

This section is devoted to the description and the analysis of a problem which we will use as a sort of "problem at infinity" for our original equation.

We first define a functional $Q_{\infty}: H_{\text {rad }}^{1}(\Omega) \backslash\{0\} \rightarrow \mathbf{R}$ as

$$
Q_{\infty}(u)=\frac{\|u\|^{2}}{\|u\|_{\infty}^{2}}
$$

and note that it is well defined because of the compact embedding of $H_{\text {rad }}^{1}(\Omega)$ into $L^{\infty}(\Omega)$.

Next, we let $\xi$ be a point of global maximum for the function $\varphi$ on $[a, b]$; by the results of the previous section, $\xi \in(a, b)$. We set

$$
\Omega^{-}=\{x \in \Omega|a<| x \mid<\xi\} \text { and } \Omega^{+}=\{x \in \Omega|\xi<| x \mid<b\},
$$


and we define the sets

$$
X_{+}=\left\{u \in H_{\text {rad }}^{1}(\Omega) \mid\|u\|_{L^{\infty}\left(\Omega^{-}\right)}<\|u\|_{L^{\infty}\left(\Omega^{+}\right)}\right\}
$$

and

$$
X_{-}=\left\{u \in H_{\text {rad }}^{1}(\Omega) \mid\|u\|_{L^{\infty}\left(\Omega^{-}\right)}>\|u\|_{L^{\infty}\left(\Omega^{+}\right)}\right\} .
$$

Notice that $X_{+}$and $X_{-}$are open subsets of $H_{\text {rad }}^{1}(\Omega)$, that $u \in X_{ \pm}$and $\lambda>0$ imply $\lambda u \in X_{ \pm}$, and finally that

$$
\partial X_{+}=\partial X_{-}=\left\{u \in H_{r a d}^{1}(\Omega) \mid\|u\|_{L^{\infty}\left(\Omega^{-}\right)}=\|u\|_{L^{\infty}\left(\Omega^{+}\right)}\right\} .
$$

Remark 3.1. Since $u \in X_{ \pm}$if and only if $|u| \in X_{ \pm}$and $Q(|u|)=Q(u)$ for every $u \in H_{\text {rad }}^{1}(\Omega)$, from now on we always assume that we deal with nonnegative functions.

The main result of this section is the following.

Proposition 3.2. We have

$$
\inf _{u \in X_{ \pm}} Q_{\infty}(u)<\inf _{u \in \partial X_{ \pm}} Q_{\infty}(u) .
$$

The proof will be carried out for the "+" case (the other one being analogous). We argue by contradiction, assuming that

$$
\inf _{u \in X_{+}} Q_{\infty}(u)=\inf _{u \in \partial X_{+}} Q_{\infty}(u)=: c_{+} \cdot
$$

In this case it is easy to check that $c_{+}$is attained by a (nonnegative) function $u$ that lies in $\partial X_{+}$and that we can normalize by requiring $\|u\|_{\infty}=1$. Therefore we have

$$
1=\|u\|_{\infty}=\|u\|_{L^{\infty}\left(\Omega^{-}\right)}=\|u\|_{L^{\infty}\left(\Omega^{+}\right)}
$$

This equality, the normalization and the continuity of $u$ say that there exist

$$
r^{-} \in[a, \xi] \text { and } r^{+} \in[\xi, b] \quad \text { such that } \quad u\left(r^{ \pm}\right)=1
$$

Lemma 3.3. There results

$$
r^{-}=r^{+}=\xi
$$

Proof. Assume that $r^{-}<r^{+}$. We first claim that $u$ cannot be constant on the interval $\left[r^{-}, r^{+}\right]$. Indeed, if $u \equiv 1$ on $\left[r^{-}, r^{+}\right]$, we take a function $v \in C_{0}^{1}\left(r^{-}, r^{+}\right)$ such that $v(r) \leq 0$, and $v \not \equiv 0$ and then, for every $\varepsilon$ positive and small we have

- $u(r)+\varepsilon v(r) \leq u(r)$ for all $r \in[a, b]$,

- $u\left(r^{ \pm}\right)+\varepsilon v\left(r^{ \pm}\right)=u\left(r^{ \pm}\right)=1$,

which show that $\|u+\varepsilon v\|_{\infty}=1$ and $u+\varepsilon v \in \partial X_{+}$. 
We now compute

$$
\begin{aligned}
Q_{\infty}(u+\varepsilon v)= & \|u\|^{2}+\varepsilon^{2}\|v\|^{2}+2 \varepsilon\langle u, v\rangle=\|u\|^{2}+\varepsilon^{2}\|v\|^{2} \\
& +2 \omega \varepsilon\left(\int_{r^{-}}^{r^{+}} u^{\prime} v^{\prime} r^{N-1} d r+\int_{r^{-}}^{r^{+}} u v r^{N-1} d r\right) \\
= & \|u\|^{2}+\varepsilon^{2}\|v\|^{2}+2 \omega \varepsilon \int_{r^{-}}^{r^{+}} v r^{N-1} d r<\|u\|^{2}
\end{aligned}
$$

for every $\varepsilon>0$ small enough, since $v$ is negative.

Therefore no $u$ constantly equal to one on $\left[r^{-}, r^{+}\right]$can attain $c_{+}$, and the claim is proved.

We now prove that $r^{-}=r^{+}$. To see this we note that if $r^{-}<r^{+}$, then, since $u$ is not constant on $\left[r^{-}, r^{+}\right]$, there exists a global minimum point $r_{0}$ for $u$ restricted to $\left[r^{-}, r^{+}\right]$. Of course $u\left(r_{0}\right)<1$. Consider the function

$$
\bar{u}(r)= \begin{cases}\min \left(u(r), u\left(r_{0}\right)\right) & \text { if } r \in\left[a, r_{0}\right] \\ u(r) & \text { if } r \in\left(r_{0}, b\right] .\end{cases}
$$

We have

- $u \in H_{\text {rad }}^{1}(\Omega)$,

- $\|\bar{u}\|_{L^{\infty}\left(\Omega^{-}\right)} \leq 1$,

- $\|\bar{u}\|_{L^{\infty}\left(\Omega^{+}\right)}=\bar{u}\left(r^{+}\right)=u\left(r^{+}\right)=1$,

and therefore $\bar{u} \in X_{+} \cup \partial X_{+}$.

The set

$$
A=\left\{r<r_{0} \mid \bar{u}(r)<u(r)\right\}
$$

contains $r^{-}$, and since $u$ and $\bar{u}$ are continuous, its measure is positive. Note also that $\left|\bar{u}^{\prime}(r)\right|=0 \leq\left|u^{\prime}(r)\right|$ almost everywhere on $A$.

Then

$$
\begin{aligned}
Q_{\infty}(\bar{u}) & =\|\bar{u}\|^{2}=\omega \int_{A}\left(\left|\bar{u}^{\prime}\right|^{2}+\bar{u}^{2}\right) r^{N-1} d r+\omega \int_{[a, b] \backslash A}\left(\left|u^{\prime}\right|^{2}+u^{2}\right) r^{N-1} d r \\
& <\omega \int_{A}\left(\left|u^{\prime}\right|^{2}+u^{2}\right) r^{N-1} d r+\omega \int_{[a, b] \backslash A}\left(\left|u^{\prime}\right|^{2}+u^{2}\right) r^{N-1} d r \\
& =\|u\|^{2}=Q_{\infty}(u) .
\end{aligned}
$$

Therefore

$$
\inf _{X_{+} \cup \partial X_{+}} Q_{\infty} \leq Q_{\infty}(\bar{u})<Q_{\infty}(u)=c_{+}=\inf _{X_{+} \cup \partial X_{+}} Q_{\infty},
$$

the last equality following from (19). This is the contradiction proving that $r^{-}=r^{+}$.

Since this holds for every $r^{-} \leq \xi \leq r^{+}$where $u$ attains its maximum, we conclude that there can be only one such point. This point must be $\xi$ because $u \in \partial X_{+}$.

End of the proof of Proposition 3.2. Any function $u$ that minimizes $Q_{\infty}$ on $\partial X_{+}$satisfies, after normalization,

$$
\|u\|_{L^{\infty}\left(\Omega^{-}\right)}=\|u\|_{L^{\infty}\left(\Omega^{+}\right)}=u(\xi)=1
$$


and $u(r)<1$ for all $r \neq \xi$. Therefore

$$
c_{+}=Q_{\infty}(u)=\|u\|^{2}=\frac{\|u\|^{2}}{u(\xi)^{2}} \geq \min _{v \in H_{\xi}} \frac{\|v\|^{2}}{v(\xi)^{2}}=\varphi(\xi),
$$

by the definition of the function $\varphi$ (Definition 2.2).

Now take $w \in H_{\text {rad }}^{1}(\Omega)$ such that

$$
\|w\|_{\infty}=1 \quad \text { and } \quad Q_{\infty}(w)=\|w\|^{2}=\varphi(b) ;
$$

such function exists by Lemma 5 . We know that $w$ is strictly increasing and that $w(b)=1$, so that

$$
\|w\|_{L^{\infty}\left(\Omega^{-}\right)}<\|w\|_{L^{\infty}\left(\Omega^{+}\right)}=1,
$$

namely, $w \in X_{+}$. But since $\xi$ is a global maximum for $\varphi$, by (20),

$$
c_{+} \geq \varphi(\xi)>\varphi(b)=Q_{\infty}(w) \geq \inf _{v \in X_{+}} Q_{\infty}(v)=c_{+},
$$

a contradiction that comes from assuming (19). The proof is complete.

Remark 3.4. Since the infimum of $Q_{\infty}$ on $X_{+} \cup \partial X_{+}$is attained, Proposition 3.2 shows that it is attained inside $X_{+}$. The same holds for $X_{-}$; we can rephrase Proposition 3.2 as

$$
\min _{u \in X_{ \pm}} Q_{\infty}(u)<\min _{u \in \partial X_{ \pm}} Q_{\infty}(u) .
$$

\section{The main existence results}

In this section we prove Theorem 1.1.

We begin by an approximation result, which we will use to show that the functional associated to Problem (1) is appropriately close to $Q_{\infty}$. First, we consider a slight variant of a classical result, that we prove for completeness.

Lemma 4.1. Let $g \in L_{\text {rad }}^{1}(\Omega)$ be positive almost everywhere in $\Omega$. Let $\left(u_{k}\right)_{k \in \mathbf{N}} \subset$ $H_{\text {rad }}^{1}(\Omega)$ be a sequence such that

$$
u_{k} \rightarrow u \quad \text { weakly in } H_{\text {rad }}^{1}(\Omega) \text { as } k \rightarrow \infty \text {. }
$$

Then, for every sequence $\left(p_{k}\right)_{k \in \mathbf{N}}$ of positive real numbers such that $p_{k} \rightarrow \infty$ as $k \rightarrow \infty$, there results

$$
\left(\int_{\Omega} g\left|u_{k}\right|^{p_{k}} d x\right)^{1 / p_{k}} \rightarrow\|u\|_{\infty} \quad \text { as } \quad k \rightarrow \infty .
$$

Proof. If $u \equiv 0$ it is trivial because

$$
\left(\int_{\Omega} g\left|u_{k}\right|^{p_{k}} d x\right)^{1 / p_{k}} \leq\|g\|_{1}^{1 / p_{k}}\left\|u_{k}\right\|_{\infty} \rightarrow 0
$$

by the compactness of the embedding of $H_{\text {rad }}^{1}(\Omega)$ into $L^{\infty}(\Omega)$.

Hence we may assume from now on that $u \neq \equiv 0$. We write

$$
\left(\int_{\Omega} g\left|u_{k}\right|^{p_{k}} d x\right)^{1 / p_{k}}=\left\|g^{1 / p_{k}} u_{k}\right\|_{p_{k}}
$$


and we observe that

$$
\left|\left\|g^{1 / p_{k}} u_{k}\right\|_{p_{k}}-\left\|g^{1 / p_{k}} u\right\|_{p_{k}}\right| \leq\left\|g^{1 / p_{k}}\left(u_{k}-u\right)\right\|_{p_{k}}=o(1)
$$

as $k \rightarrow \infty$ since $u_{k} \rightarrow u$ in $L^{\infty}(\Omega)$.

Next, obviously

$$
\left\|g^{1 / p_{k}} u\right\|_{p_{k}} \leq\|g\|_{1}^{1 / p_{k}}\|u\|_{\infty}=\|u\|_{\infty}+o(1)
$$

so that we only have to obtain an estimate from below. To get this, we fix $\varepsilon>0$ such that $\varepsilon<\|u\|_{\infty}$, which is possible since $u \not \equiv 0$, and we set

$$
\Omega_{\varepsilon}=\left\{x \in \Omega|| u(x) \mid \geq\|u\|_{\infty}-\varepsilon\right\} .
$$

Then

$$
\left\|g^{1 / p_{k}} u\right\|_{p_{k}} \geq\left(\int_{\Omega_{\varepsilon}} g|u|^{p_{k}} d x\right)^{1 / p_{k}} \geq\left(\int_{\Omega_{\varepsilon}} g d x\right)^{1 / p_{k}}\left(\|u\|_{\infty}-\varepsilon\right),
$$

which shows that as $k \rightarrow \infty$,

$$
\left\|g^{1 / p_{k}} u\right\|_{p_{k}} \geq\|u\|_{\infty}-\varepsilon+o(1) .
$$

Thus,

$$
\|u\|_{\infty}-\varepsilon+o(1) \leq\left\|g^{1 / p_{k}} u\right\|_{p_{k}} \leq\|u\|_{\infty}+o(1) .
$$

Since this holds for every $\varepsilon$ positive and small, we obtain

$$
\left\|g^{1 / p_{k}} u\right\|_{p_{k}} \rightarrow\|u\|_{\infty}
$$

as $k \rightarrow \infty$. From (21) we conclude that

$$
\left\|g^{1 / p_{k}} u_{p_{k}}\right\|_{p_{k}} \rightarrow\|u\|_{\infty}
$$

as $k \rightarrow \infty$.

In the next lemma we show that some uniformity can forced into the previous statement.

Lemma 4.2. Let $g \in L_{\text {rad }}^{1}(\Omega)$ be positive almost everywhere in $\Omega$. Then, as $p \rightarrow \infty$,

$$
\left(\int_{\Omega} g|u|^{p} d x\right)^{1 / p}=\|u\|_{\infty}+o(1)
$$

uniformly on bounded subsets of $H_{\text {rad }}^{1}(\Omega)$.

Proof. Let $U$ be a bounded subset of $H_{\text {rad }}^{1}(\Omega)$. We show that

$$
\lim _{p \rightarrow \infty} \sup _{u \in U}\left|\left(\int_{\Omega} g|u|^{p} d x\right)^{1 / p}-\|u\|_{\infty}\right|=0 \text {. }
$$

Assume this is false; then there exist $\delta>0$, a sequence $p_{k} \rightarrow \infty$, and a sequence $u_{k} \in U$ such that

$$
\left|\left(\int_{\Omega} g\left|u_{k}\right|^{p_{k}} d x\right)^{1 / p_{k}}-\left\|u_{k}\right\|_{\infty}\right| \geq \delta
$$


for every $k$. Since $u_{k}$ is bounded in $H_{r a d}^{1}(\Omega)$, there exists a subsequence, still denoted $u_{k}$, such that

$$
u_{k} \rightarrow u \quad \text { in } H_{r a d}^{1}(\Omega) \text { and } \quad u_{k} \rightarrow u \quad \text { in } \quad L^{\infty}(\Omega) .
$$

Then, as $k \rightarrow \infty$,

$$
\begin{aligned}
\delta & \leq\left|\left(\int_{\Omega} g\left|u_{k}\right|^{p_{k}} d x\right)^{1 / p_{k}}-\left\|u_{k}\right\|_{\infty}\right| \\
& \leq\left|\left(\int_{\Omega} g\left|u_{k}\right|^{p_{k}} d x\right)^{1 / p_{k}}-\|u\|_{\infty}\right|+\left|\|u\|_{\infty}-\left\|u_{k}\right\|_{\infty}\right|=o(1)
\end{aligned}
$$

by the preceding lemma. This contradiction completes the proof.

We are now ready to prove the existence results. To this aim we consider the functional $Q_{p}: H_{\text {rad }}^{1}(\Omega) \backslash\{0\} \rightarrow \mathbf{R}$ defined by

$$
Q_{p}(u)=\frac{\|u\|^{2}}{\left(\int_{\Omega} g(|x|)|u|^{p} d x\right)^{2 / p}} .
$$

By homogeneity, critical points of $Q_{p}$ restricted to the sphere

$$
\mathcal{S}=\left\{u \in H_{\text {rad }}^{1}(\Omega) \mid\|u\|=1\right\}
$$

are free critical points of $Q_{p}$.

The preceding lemma says that

$$
Q_{p}(u)=Q_{\infty}(u)+o(1)
$$

uniformly on $\mathcal{S}$ as $p \rightarrow \infty$. Let $X_{+}$and $X_{-}$be the sets introduced in (16) and (17). Then, by Proposition 3.2 and (22), for all $p$ large enough, we have

$$
\inf _{u \in X_{ \pm} \cap \mathcal{S}} Q_{p}(u)<\inf _{u \in \partial X_{ \pm} \cap \mathcal{S}} Q_{p}(u)
$$

Proof of Theorem 1.1. The numbers

$$
\inf _{u \in X_{+} \cap \mathcal{S}} Q_{p}(u) \quad \text { and } \quad \inf _{u \in X_{-} \cap \mathcal{S}} Q_{p}(u)
$$

are attained in $\left(X_{+} \cup \partial X_{+}\right) \cap \mathcal{S}$ (and in $\left(X_{-} \cup \partial X_{-}\right) \cap \mathcal{S}$, respectively) once again because of the compactness of the embedding $H_{\text {rad }}^{1}(\Omega) \hookrightarrow L^{\infty}(\Omega)$. The inequality (23) shows that for every $p$ large enough, the above quantities are attained by two functions $u_{+}$and $u_{-}$respectively inside $X_{+} \cap \mathcal{S}$ and $X_{-} \cap \mathcal{S}$. Since these are open sets in $\mathcal{S}$, the minimizers $u_{+}$and $u_{-}$are critical points of $Q_{p}$ over $\mathcal{S}$, and hence over $H_{\text {rad }}^{1}(\Omega)$. With the usual procedures, suitable multiples of the $u_{+}$and $u_{-}$give rise to two solutions of problem (1). These solutions are of course different since $X_{+} \cap X_{-}=\emptyset$.

We now construct a third solution by a minimax procedure on $\mathcal{S}$.

Remark 4.3. Since $Q_{p}$ is homogeneous, if $u \in \mathcal{S}$, then $\nabla Q_{p}(u)$ is tangent to $\mathcal{S}$ at $u$. Therefore $\mathcal{S}$ is invariant for the standard gradient flow generated by $\nabla Q_{p}(u)$. This means that the usual deformations arguments work for $Q_{p}$ restricted to $\mathcal{S}$. 
In what follows we always assume that $p$ is sufficiently large. Let $u_{ \pm} \in$ $X_{ \pm} \cap S$ be the minimizers for $Q_{p}$ found above, and set

$$
\Gamma=\left\{\gamma \in C([0,1] ; \mathcal{S}) \mid \gamma(0)=u_{-}, \gamma(1)=u_{+}\right\}
$$

and

$$
c=\inf _{\gamma \in \Gamma} \max _{s \in[0,1]} Q_{p}(\gamma(s)) .
$$

Note that for every $\gamma \in \Gamma$,

$$
\|\gamma(0)\|_{L^{\infty}\left(\Omega^{-}\right)}-\|\gamma(0)\|_{L^{\infty}\left(\Omega^{+}\right)}=\left\|u_{-}\right\|_{L^{\infty}\left(\Omega^{-}\right)}-\left\|u_{-}\right\|_{L^{\infty}\left(\Omega^{+}\right)}>0,
$$

and likewise,

$$
\|\gamma(1)\|_{L^{\infty}\left(\Omega^{-}\right)}-\|\gamma(1)\|_{L^{\infty}\left(\Omega^{+}\right)}<0 .
$$

Therefore, for every $\gamma \in \Gamma$ there exists $s_{\gamma} \in(0,1)$ such that

$$
\left\|\gamma\left(s_{\gamma}\right)\right\|_{L^{\infty}\left(\Omega^{-}\right)}=\left\|\gamma\left(s_{\gamma}\right)\right\|_{L^{\infty}\left(\Omega^{+}\right)},
$$

namely

$$
\gamma\left(s_{\gamma}\right) \in \partial X_{-}=\partial X_{+}
$$

Hence,

$$
\begin{aligned}
c & =\inf _{\gamma \in \Gamma} \max _{s \in[0,1]} Q_{p}(\gamma(s)) \geq \inf _{\gamma \in \Gamma} Q_{p}\left(\gamma\left(s_{\gamma}\right)\right) \geq \inf _{u \in \partial X_{-}} Q_{p}(u) \\
& >\max \left\{Q_{p}\left(u_{-}\right), Q_{p}\left(u_{+}\right)\right\}=\max \left\{Q_{p}(\gamma(0)), Q_{p}(\gamma(1))\right\} .
\end{aligned}
$$

The strict inequality holds for $p$ large because of (23).

Since the functional $Q_{p}$ trivially satisfies the Palais-Smale condition, the Mountain Pass Theorem can be invoked to say that $Q_{p}$ has a third critical point on $\mathcal{S}$ at a level $\geq c$. This critical point thus gives rise, via standard techniques, to a third solution of problem (1), different from $u_{+}$and $u_{-}$.

\section{The autonomous case}

We now complete the existence results by turning to the autonomous case, namely we suppose from now on that $g \equiv 1$. In this case of course, the problem

$$
\begin{cases}-\Delta u+u=u^{p-1} & \text { in } \Omega \\ u>0 & \text { in } \Omega \\ \frac{\partial u}{\partial \nu}=0 & \text { on } \partial \Omega\end{cases}
$$

admits the constant solution $u \equiv 1$ for every $p$. 
The application of Theorem 1.1 shows that for $p$ large problem (24) admits two non constant solutions obtained by minimizing the functional

$$
Q_{p}(u)=\frac{\|u\|^{2}}{\left(\int_{\Omega}|u|^{p} d x\right)^{2 / p}}
$$

(the same functional as above, but with $g \equiv 1$ ) over $X_{-}$and $X_{+}$. Notice that the fact that the minimizers belong to $X_{-}$and $X_{+}$rules out the constant solution.

Our aim is to show that the minimax procedure just carried out yields a further nonconstant solution.

To see this we are going to modify slightly the minimax argument to obtain an estimate from above on the minimax level.

To start with, we work out an inequality for the mountain pass level of the limit problem, namely for the functional $Q_{\infty}$ defined in (15).

Let $u_{a} \in X_{-} \cap \mathcal{S}$ and $u_{b} \in X_{+} \cap \mathcal{S}$ be the unique positive functions in $\mathcal{S}$ that attain $\varphi(a)$ and $\varphi(b)$, respectively. These are just the minimizers found in Sect. 2 divided by their $H^{1}$ norm.

Consider the class

$$
\Gamma_{a b}=\left\{\gamma \in C([0,1] ; \mathcal{S}) \mid \gamma(0)=u_{a}, \gamma(1)=u_{b}\right\}
$$

and the level

$$
c_{\infty}=\inf _{\gamma \in \Gamma_{a b}} \max _{s \in[0,1]} Q_{\infty}(\gamma(s)) .
$$

As above, for every $\gamma \in \Gamma_{a b}$,

$$
\|\gamma(0)\|_{L^{\infty}\left(\Omega^{-}\right)}-\|\gamma(0)\|_{L^{\infty}\left(\Omega^{+}\right)}=\left\|u_{a}\right\|_{L^{\infty}\left(\Omega^{-}\right)}-\left\|u_{a}\right\|_{L^{\infty}\left(\Omega^{+}\right)}>0,
$$

and

$$
\|\gamma(1)\|_{L^{\infty}\left(\Omega^{-}\right)}-\|\gamma(1)\|_{L^{\infty}\left(\Omega^{+}\right)}<0 .
$$

Again we find that for every $\gamma \in \Gamma_{a b}$ there exists $s_{\gamma} \in(0,1)$ such that

$$
\gamma\left(s_{\gamma}\right) \in \partial X_{-}=\partial X_{+}
$$

This shows that

$$
\begin{aligned}
c_{\infty} & \geq \inf _{\gamma \in \Gamma_{a b}} \max _{s \in[0,1]} Q_{\infty}(\gamma(s)) \geq \inf _{\gamma \in \Gamma_{a b}} Q_{\infty}\left(\gamma\left(s_{\gamma}\right)\right) \geq \inf _{u \in \partial X_{-}} Q_{\infty}(u) \\
& >\max \left\{Q_{\infty}\left(u_{a}\right), Q_{\infty}\left(u_{b}\right)\right\}=\max \left\{Q_{\infty}(\gamma(0)), Q_{\infty}(\gamma(1))\right\}
\end{aligned}
$$

Next we estimate $c_{\infty}$ from above, constructing a special path $\gamma_{a b} \in \Gamma_{a b}$.

For $\rho \in[a, b]$, let $u_{\rho} \in H_{\rho} \cap \mathcal{S}$ be the unique positive function in $\mathcal{S}$ that attains $\varphi(\rho)$. We know that $u_{\rho}$ depends continuously on $\rho$. We define $\gamma_{a b} \in C([0,1] ; \mathcal{S})$ by

$$
\gamma_{a b}(s)=u_{s b+(1-s) a} .
$$

Clearly $\gamma_{a b}$ is continuous and $\gamma_{a b}(0)=u_{a}, \gamma_{a b}(1)=u_{b}$, so that $\gamma_{a b} \in \Gamma_{a b}$. 
Recall that $\xi$ denotes any point in $(a, b)$ where the function $\varphi$ attains its global maximum. Then

$$
\begin{aligned}
c_{\infty} & \leq \max _{s \in[0,1]} Q_{\infty}\left(\gamma_{a b}(s)\right)=\max _{s \in[0,1]} Q_{\infty}\left(u_{s b+(1-s) a}\right) \\
& =\max _{s \in[0,1]} \varphi(s b+(1-s) a)=\varphi(\xi) .
\end{aligned}
$$

Now the constant function $v \equiv|\Omega|^{-1 / 2}$ is in $\mathcal{S}$ and belongs to every $H_{\rho}$, and in particular to $H_{\xi}$. Then it must be

$$
\varphi(\xi)<Q_{\infty}(v)=|\Omega|,
$$

since in $H_{\xi} \cap \mathcal{S}$ there is a unique positive $u_{\xi}$ that attains $\varphi(\xi)$, and this function cannot be $v$ because it is strictly increasing on $[a, \xi]$ by the results of Sect. 2 .

Thus, there exists $\varepsilon_{0}>0$ such that

$$
c_{\infty} \leq \varphi(\xi) \leq Q_{\infty}(v)-2 \varepsilon_{0}=|\Omega|-2 \varepsilon_{0} .
$$

To conclude we just have to notice that if we set

$$
c_{p}=\inf _{\gamma \in \Gamma_{a b}} \max _{s \in[0,1]} Q_{p}(\gamma(s)),
$$

then for all $p$ large enough, by Lemma 4.2, we still have the strict inequality (uniform in $p$ )

$$
c_{p}>\max \left\{Q_{p}\left(u_{a}\right), Q_{p}\left(u_{b}\right)\right\},
$$

which means (since the Palais-Smale condition holds) that there exists a critical point $w_{p}$ for $Q_{p}$ at level $c_{p}$. For all $p$ large enough we have

$$
c_{p} \leq c_{\infty}+\varepsilon_{0},
$$

so that

$$
c_{p} \leq|\Omega|-\varepsilon_{0},
$$

with $\varepsilon_{0}$ independent of $p$, of course. The constant function $v \equiv|\Omega|^{-1 / 2}$ is in $\mathcal{S}$ and satisfies

$$
Q_{p}(v)=|\Omega|^{1-2 / p}=|\Omega|+o(1)
$$

as $p \rightarrow \infty$. Therefore, for all $p$ large enough,

$$
Q_{p}\left(w_{p}\right)=c_{p} \leq|\Omega|-\varepsilon_{0}<Q_{p}(v),
$$

and hence $w_{p}$ is not constant.

We also have that $w_{p}$ is different, for $p$ large, from the two local minimizers $u_{ \pm} \in X_{ \pm} \cap \mathcal{S}$ found earlier because, for example, $Q_{p}\left(u_{-}\right)=Q_{p}\left(u_{a}\right)+o(1)$ as $p \rightarrow \infty$, and then the strict inequality $(27)$ shows that $Q_{p}\left(w_{p}\right)>Q_{p}\left(u_{-}\right)$ for all $p$ large, and likewise for $u_{+}$.

From $w_{p}$ one constructs a positive solution of problem (4) with standard arguments.

This concludes the proof. 
Note added in proof. After the paper was submitted for publication, our attention was drawn to [5] where the authors consider a problem, in the ball, similar to ours. Though the results are not totally comparable, both hold in the case of constant coefficients. It is therefore natural to ask whether our third positive solution (the one obtained at a mountain pass level) can be found on a ball.

\section{References}

[1] Bahri, A., Coron, J.-M.: On a nonlinear elliptic equation involving the critical Sobolev exponent: the effect of the topology of the domain. Commun. Pure Appl. Math. 41(3), 253-294 (1988)

[2] Barutello, V., Secchi, S., Serra, E.: A note on the radial solutions for the supercritical Hnon equation. J. Math. Anal. Appl. 341(1), 720-728 (2008)

[3] Brezis, H., Nirenberg, L.: Positive solutions of nonlinear elliptic equations involving critical Sobolev exponents. Commun. Pure Appl. Math. 36(4), 437-477 (1983)

[4] Grossi, M.: Asymptotic behaviour of the Kazdan-Warner solution in the annulus. J. Diff. Eqns. 223, 96-111 (2006)

[5] Grossi, M., Noris, B.: Positive constrained minimizers for supercritical problems in the ball. arXiv: 1006.5360v11 (2010)

[6] Kazdan, J.L., Warner, F.W.: Remarks on some quasilinear elliptic equations. Commun. Pure Appl. Math. 28(5), 567-597 (1975)

[7] Lin, C.S., Ni, W.-M.: On the diffusion coefficient of a semilinear Neumann problem. Calculus of Variations and Partial Differential Equations (Trento, 1986). Lecture Notes in Mathematics, vol 1340, pp. 160-174. Springer, Berlin (1988)

[8] Ni, W.-M., Ming, W.: On the positive radial solutions of some semilinear elliptic equations on $R^{n}$. Appl. Math. Optim. 9(4), 373-380 (1983)

[9] Ni, W.-M., Nussbaum, R.D.: Uniqueness and nonuniqueness for positive radial solutions of $\Delta u+f(u, r)=0$. Commun. Pure Appl. Math. 38(1), 67-108 (1985)

[10] Passaseo, D.: Nonexistence results for elliptic problems with supercritical nonlinearity in nontrivial domains. J. Funct. Anal. 114(1), 97-105 (1993)

[11] Pohožaev, S.: Eigenfunctions of the equation $\Delta u+\lambda f(u)=0$. Sov. Math. Dokl. 6, 1408-1411 (1965)

[12] Serra, E., Tilli, P.: Monotonicity constraints and supercritical Neumann problems. Preprint (2009) 
D. Bonheure

Département de Mathématique,

Université Libre de Bruxelles,

ULB, CP214 Boulevard du Triomphe,

1050 Brussels,

Belgium

E. Serra

Dipartimento di Matematica, Politecnico di Torino

Corso Duca degli Abruzzi, 24, 10129 Turin,

Italy

e-mail: enrico.serra@polito.it

Received: 12 July 2010.

Revised: 14 October 2010.

Accepted: 05 November 2010. 